\title{
Problematisch: Analgetika vor dem Marathon
}

\section{Die Einnahme von Analgetika vor extremen Ausdauerbelastungen verringert nicht die Schmerzen, geht aber mit einer Erhöhung der Inzidenz kardiovaskulärer, gastrointestinaler und renaler Schäden einher.}

— Die Teilnehmerzahl der großen Marathonläufe wie in Boston oder London geht mittlerweile in die Zehntausende. Marathon laufen ist „in“ und auch Triathlon findet eine wachsende Zahl von Aficionados. Unter Insidern ist es mittlerweile üblich, vor einem Marathon NSAID einzunehmen, um die auf der Strecke zweifellos auftretenden Schmerzen an verschiedenen Körperteilen erträglicher zu gestalten.

Während des Bonn-Marathons 2010 wurden an die 7048 Teilnehmer Fragebögen verteilt, von denen 3913 ausgewertet werden konnten. $49 \%$ der Teilnehmer am Lauf gaben an, vorher Analgetika eingenommen zu haben. In 54\% handelte es sich dabei um OTC-Präpa- rate ohne Verschreibung. Frauen nahmen zu $61 \%$, Männer nur zu $42 \%$ Analgetika ein. Das am häufigsten gebrauchte Präparat war Diclofenac, das von $47 \%$ der Kohorte mit Analgetika eingenommen wurde. An zweiter Stelle stand Ibuprofen, wobei besonders bemerkenswert erscheint, dass $43 \%$ mit einer Dosis von $800 \mathrm{mg}$ und mehr das Doppelte der empfohlenen Einzeldosis einnahmen. Weitere gebräuchliche Analgetika waren ASS, Paracetamol, Celecoxib, Dipyron, Etoricoxib, Meloxicam oder Naproxen.

43\% der Teilnehmer gaben an, dass sie nicht über die Risiken von Analgetika in Verbindung mit Ausdauersport informiert waren. Die Inzidenz berichteter unerwünschter Wirkungen lag bei den Marathonläufern mit Analgetikaeinnahme fünffach höher als bei den Läufern ohne diese Maßnahme. Diese Inzidenz stieg mit wachsender Dosis signifikant an. Von den neun Personen, die ins Krankenhaus aufgenommen wurden, hatten alle Analgetika eingenommen.
Drei Athleten berichteten über eine Anurie/Oligurie, vier wurden wegen einer gastrointestinalen Blutung stationär aufgenommen, zwei Teilnehmer klagten über Brustschmerzen, pektanginöse Beschwerden und Arrhythmien. Die diesbezügliche Dunkelziffer dürfte erheblich sein.

\footnotetext{
- M. Küster et al.

(Korres.: kay.brune@pharmakologie.med. uni-erlangen.de): Consumption of analgesics before a marathon and the incidence of cardiovascular, gatrointestinal and renal problems: a cohort study. BMJ Open 2013;3: e002090
}

\section{Kommentar}

Die Gefahren einer Einnahme von Analgetika vor Marathonläufen scheint nicht allen Teilnehmern bewusst zu sein. Vom medizinischen Standpunkt her sind Marathonläufe als nicht gesund zu bezeichnen. Mit Analgetika werden sie zum Risiko.

H. S. FÜESSL -

\section{Einmalige Liveshow}

- Ein 52-jähriger Mann mit einem bekannten hereditären Angioödem aufgrund eines C1-Esterase-Inhibitormangels unterzog sich wegen einer Divertikulose einer Kolonteilresek-
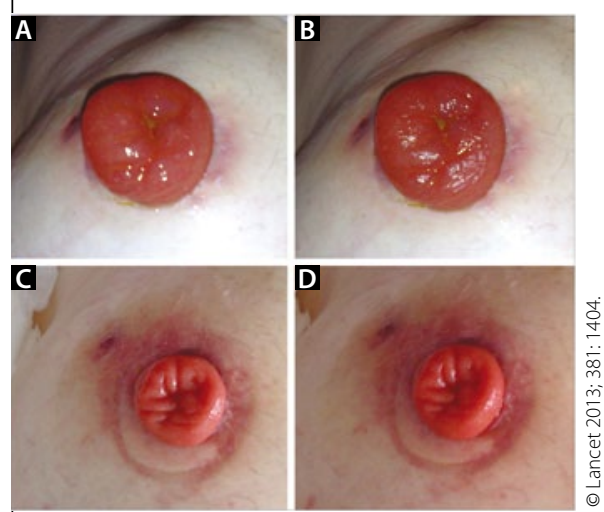

Angioödem (A) und Rückgang der Mukosaschwellung (C-D) unter Therapie. tion. Postoperativ kam es aufgrund einer Anastomoseninsuffizienz zu einer Sepsis, die eine Kolostomie erforderlich machte.

Drei Monate nach der Operation traten kolikartige Bauchschmerzen und Erbrechen auf. Dabei bemerkte man eine deutliche Schwellung der Mukosa im Bereich der Kolostomie (Abb. A). Nach Therapie mit dem C1Inhibitor-Konzentrat Berinert ${ }^{\circledR}$ gingen die Symptome allmählich im Lauf einer Stunde zurück und waren zwölf Stunden später komplett verschwunden. Der Rückgang der Mukosaschwellung verlief langsamer und war erst nach 48 Stunden nicht mehr feststellbar (Abb. B bis D).
- H. Farkas und D. Csuka

(Korres.: farkash@kut.sote.hu): An abdominal attack of hereditary angio-oedema. Lancet 2013;381:1404.

\section{Kommentar}

Beim hereditären Angioödem kann es auch zu intestinalen Ödemen unter dem Bild eines akuten Abdomens kommen. Typisch für das Angioödem ist freie Flüssigkeit im Peritonealraum, ein Ödem der Mukosa, das sonografisch festgestellt werden kann und eine rasche Besserung nach entsprechender Therapie. Im vorliegenden Fall bot die freiliegende Mukosa die einmalige Gelegenheit, das Angioödem und das Ansprechen der Therapie direkt zu beobachten.

H. S. FÜESSL . 